\title{
Parameter Optimization of Magnetostrictive Linear Displacement Transducer
}

\author{
Xisheng $\mathrm{Li}^{1, \mathrm{a}}$, Zhenyan $\mathrm{Wu}^{1, \mathrm{~b}}$, Xiongying $\mathrm{Shu}^{1, \mathrm{c}}$, Xuexu $\mathrm{Gao}^{2, \mathrm{~d}}$ and Mingming \\ $\mathrm{Li}^{2, \mathrm{e}}$ \\ ${ }^{1}$ School of Automation and Electrical Engineering, University of Science and Technology Beijing, \\ Beijing 100083, China \\ ${ }^{2}$ State Key Laboratory for Advanced Metals and Materials, University of Science and Technology \\ Beijing, Beijing 100083, China \\ axs@@ustb.edu.cn, ${ }^{\mathrm{b}} 757733459 @ q q . c o m,{ }^{c}$ limosine@sohu.com, ${ }^{\mathrm{d}}$ gaox@skl.ustb.edu.cn, ${ }^{\mathrm{e}}$ limingmin \\ g_0631@163.com
}

Keywords: Magnetostrictive waveguide; Linear position sensor; Torsional wave; Wiedeman effect.

\begin{abstract}
The magnetostrictive linear displacement transducer is a kind of transducer that can calculate the distance between the measured point and the receiving point by measuring the time difference between the excitation and the receipt of elastic waves. This paper describes research work in a kind of magnetostrictive linear displacement transducer based on Wiedemann effect, focusing on the enhancement of the torsional strain pulse generation with the aim of obtaining high measurement accuracy. The pulse width and amplitude of the exciting pulse current are optimized for increasing the output signal amplitude of torsional wave sensing coil. The results of experiment show that through the parameter optimization the electric reply signal is increased, the signal-to-noise ratio of sensing coil output can be improved.
\end{abstract}

\section{Introduction}

Measurement of linear displacement is one of the fundamental measurements in many industrial processes. At present, there are many kinds of linear displacement transducers. For most kinds of position detection transducers, such as potentiometers, capacitor, optical encoders, optical grating, and laser interferometers, there is a common disadvantage that they demand for strict environmental conditions due to their weak environmental adaptability. In other words, these transducers usually fail to work well, when they are used in the conditions of greasy dirt, oil, or water. For this reason, more robust sensors are wanted for linear displacement measurement.

Magnetostrictive linear displacement transducers can work in harsh industrial environment and have a long operational life since there is no contact between sensing element and cursor, offering an alternative way for linear position sensing. In general, magnetostrictive linear displacement transducers are based on Wiedemann effect. Wiedemann effect is the phenomenon of the twisting of a rod or tube due to magnetostriction when longitudinal and circumferential magnetic fields are applied simultaneously [1, 2]. Magnetostrictive linear displacement transducers calculate the distance between the measured point and the receiving point from the time-of-flight of strain wave.

Nowadays, the development of magnetostrictive linear displacement transducer is always driven by the ongoing pursuit of higher accuracy, larger measurement range, and higher reliability. In order to improve the measurement precision magnetostrictive displacement sensor, a lot of research works have been carried out. A macroscopic model of the response of magnetostrictive delay lines (MDLs) for generating longitudinal ultrasonic wave under pulsed current excitation has been established in [3]. Comparison result of theoretical curves with experimental data on a Fe78Si7B15 amorphous ribbon sample show that they are of sufficient agreement. By calculating the cross-correlation function of the output signals of piezoelectric ceramic type elastic wave sensing element located at both ends of the waveguide which are digitized by high-speed data acquisition system and peak searching for time delay estimation, displacement value is computed [4, 5]. The developed prototype 
span $1000 \mathrm{~mm}$, nonlinear error is less than $30 \mu \mathrm{m}$, repeatability can be achieved $10 \mu \mathrm{m}$. By using a multiple wire delay line, a large output signal for a given input and cross sectional area of the line is obtained [6]. Meanwhile, the influence of local inhomogeneities on the amplitude of the output signal is reduced. Physical parameters, such as the pulse current width, amplitude and the distance between the permanent and waveguide, has been optimized on a trial basis to improve the sensing coil output signal amplitude [7]. But the waveguide material used has not been given in [7]. The Dependence of the sensing coil output voltage pulse on the exciting current pulse amplitude for used waveguide material 4J50 have been conducted [8]. Wave interference method is used to increase the sensing element output signal amplitude [9-11]. By using constructive interference among undamped torsional waves that propagate along the magnetostrictive waveguide, the output signal amplitude of the sensing coil is increased [9]. Because of simplified construction by eliminating dumpers, this method can reduce costs and extend transducer life. Wave array interference method using a structure that consists of multi-pulses and multi-cursors has been adopted in [10]. By adopting bipolar excitation and single-end damper structure, wave interference method using reflected wave is realized [11]. High precision time interval measurement using CPLD is adopted to improve the measurement resolution [12]. Research works on the material composition and treatment for improving the performance of the waveguide wire have been done too [13-15]. The experimental results of former researchers show that the optimal parameters are related to the magnetostrictive waveguide $[7,8]$.

In general, sensing element output signal amplitude directly affects the transducer characteristics. As the measurement range is increased, the output signal amplitude becomes weaker due to the acoustic impedance of the waveguide. It becomes more difficult to maintain sufficient signal to noise ratio (SNR).

In this paper, physical parameter optimization of the newly developed magnetostrictive linear displacement transducer is introduced. Specially, in order to increase the sensing coil output signal amplitude, the pulse width and the amplitude of exciting current are optimized.

\section{Fundamental Principle of Magnetostrictive Linear Displacement Transducer}

Magnetostrictive linear displacement transducers are based on magnetostrictive properties that some ferromagnetic materials show when subjected to magnetic fields or deformations. Magnetostriction is a phenomenon only found in ferromagnetic materials, such as iron, nickel, cobalt and their alloys. Ferromagnetic materials placed in a magnetic field undergo microscopic distortion of the molecular structure. This physical phenomenon is called magnetostriction. In fact, magnetostrictive effect includes Joule effect and Wiedemann effect. When bringing a bar of ferromagnetic material into a magnetic field oriented in parallel to the longitudinal direction of the bar, there will be mechanical length change of the bar. This physical phenomenon is called Joule effect. The Wiedemann effect is a phenomenon that a ferromagnetic cylindrical rod or tube specimen fixed at its one end, twists around the rod or tube axis, when it is magnetized under the simultaneous action of longitudinal magnetic field, $H_{l}$, parallel to, and circular field, $H_{c}$, around the rod or tube axis.

In general, the Wiedemann effect is used by the industrial magnetostrictive linear displacement transducer. When a current pulse is applying onto the magnetostrictive waveguide, this current pulse produces a circular magnetic field perpendicular to the axis of the waveguide. At measured point, the longitudinal magnetic field generated by the permanent magnet built in cursor is superimposed with the circular magnetic field, a spiral magnetic field is formed, and the magnetostrictive waveguide is distorted elastically (Wiedemann effect). In other words, torsional strain wave is generated.

Generated torsional strain wave travels along the waveguide at constant sound speed from the measured point. The propagation velocity of the generated torsional wave $V$ can be expressed as

$$
V=\sqrt{\frac{G}{\rho}},
$$

where $G$ is the shear modulus of the waveguide, $\rho$ is the density of the waveguide. 
The torsional strain pulse is converted back to an electrical signal through the inverse magnetostrictive (Villari) effect at receiving point. Several kinds of sensing element, such as an axial coil, a piezoelectric sensing element and a coil encircling a small tape of a magnetostrictive material perpendicular to the waveguide, can be used to transform the torsional wave into an electric signal. In this paper, an axial coil is adopted as a simple structure, low-cost, non-contact signal converter. As the torsional strain pulse reaches the sensing coil, an electric pulse is generated.

According to Villari effect, at the time of occurrence of physical deformation of magnetostrictive waveguide, the magnetic field strength inside the waveguide changes, the magnetic flux pass through the torsional wave sensing coil will changes. According to Faraday's law of electromagnetic induction, the induced electromotive force $\varepsilon$ can be expressed as

$$
\varepsilon=n \frac{d \Phi}{d t}
$$

where $t$ is time, $\Phi$ is magnetic flux, $n$ is the coil turn number. Therefore, the sensing coil at the near end of magnetostrictive linear displacement transducer will output an induced electromotive force pulse. At the far end of the transducer, arrived travelling torsional strain wave is completely absorbed by damper in order to preventing reflection of the torsional wave.

The induced reply signal is amplified, and fed to a comparator in order to convert into a pulse signal. The exciting current pulse control signal and the return pulse signal are fed to the counting circuit, and the time interval $T$ is measured. The distance between the torsional wave receiving point and the measured position is calculated according to the measured time interval,

$$
L=V T \text {, }
$$

the displacement measurement is accomplished.

This is a continuous measurement process. As the position of permanent magnet changes, the new position will be detected in less than 0.01 seconds of time. The schematic diagram of magnetostrictive linear displacement transducer is shown in Fig. 1.

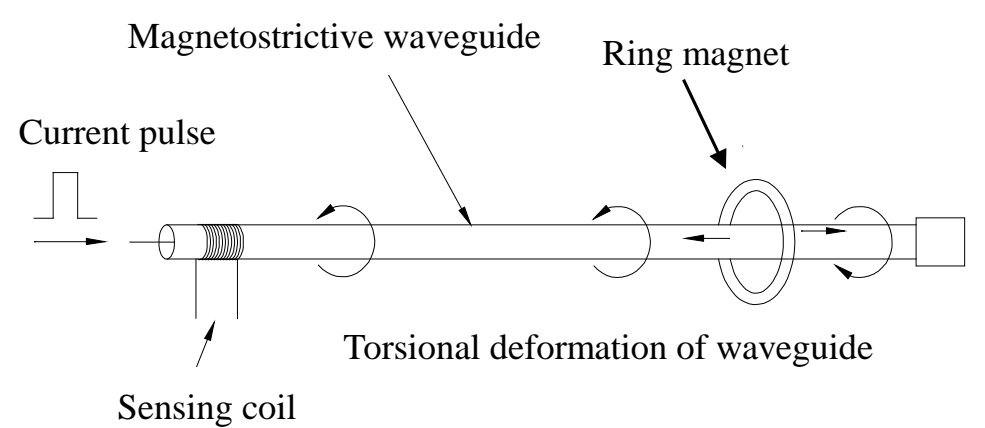

Fig. 1. Scheme of magnetostrictive linear displacement transducer

\section{Circuit Design of Magnetostrictive Linear Displacement Transducer}

The measurement circuit of developed magnetostrictive linear displacement transducer comprises a pulse generating circuit, a power amplification circuit, a signal amplifying and shaping circuit, a serial communication interface circuit, a power supply circuit and a time interval measuring circuit. Among them, the power supply circuit is responsible for each function circuitry, the serial communication interface circuit provides RS-232 interface to connect computers and other equipment in order to transfer displacement measurement data to them. A circuit block diagram shown in Fig. 2.

A control pulse signal of frequency $100 \mathrm{~Hz}, 1 \sim 20 \mu$ s adjustable pulse width is generated by the pulse generating circuit. This control pulse signal is amplified by the power amplifier circuit in order to generate the driving pulse current of amplitude up to 7.5A for magnetostrictive waveguide. When the pulse current is applied onto the magnetostrictive waveguide, the circular magnetic field generated by this pulse current will superimpose with the longitudinal magnetic field generated by 
the permanent magnet, and torsional strain wave will be generated. The torsional wave propagating to the near end is detected by the sensing coil, and a reply signal is induced. And then this induced reply signal is amplified and reshaped to produce a reply pulse. The control pulse and reply pulse are fed to the time interval measurement circuit. According the measured value of the time interval between control pulse and reply pulse, the distance between permanent magnet and sensing coil is computed.

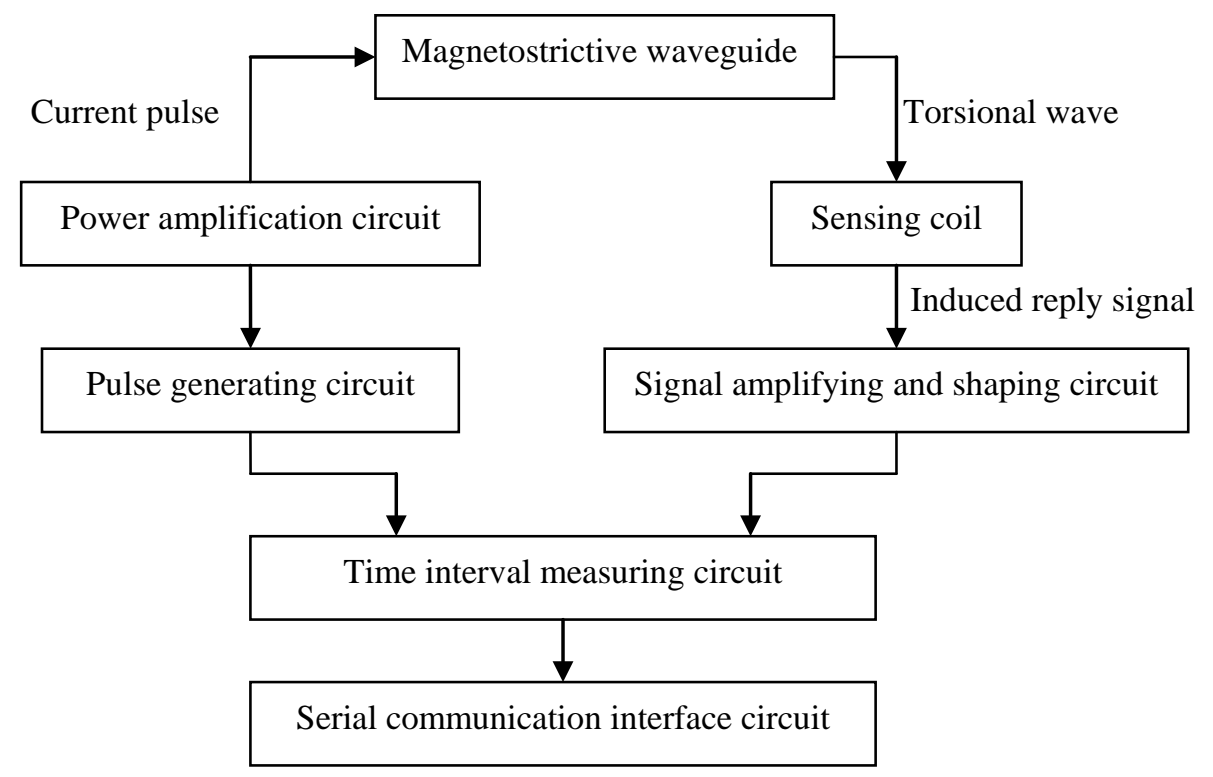

Fig. 2. Circuit block diagram of magnetostrictive linear displacement transducer

The measurement principle of time interval is shown in Fig. 3. Time interval measurement circuit takes FPGA chip as the core. In this paper, EP3C25 from Altera is chosen. In the time measurement module, the time interval to be measured is filled with high frequency pulse signal. The high-frequency pulse of frequency $250 \mathrm{MHz}$ is generated by frequency multiplication using phase-locked loop in EP3C25.

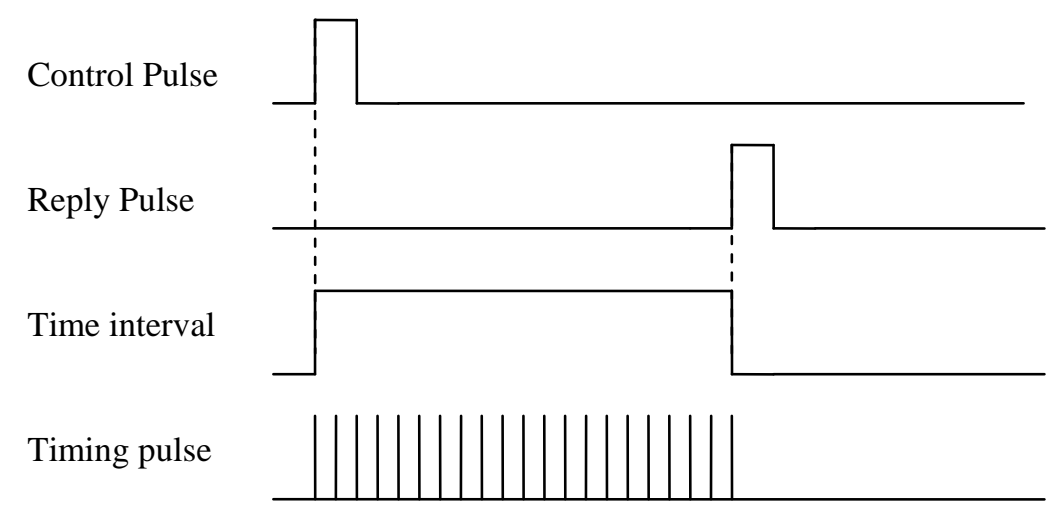

Fig. 3. The principle of time interval measurement

\section{Parameter Optimization of Magnetostrictive Linear Displacement Transducer}

The applied current pulse signal produces a circular magnetic field perpendicular to the magnetostrictive waveguide. At measured position, this circular magnetic field is superimposed to the longitudinal magnetic field produced by permanent magnet, and a spiral magnetic field is formed. Torsion deformation of magnetostrictive waveguide is produced due to spiral magnetic field, thereby a torsional wave is stimulated. According to the above analysis, to change the current pulse 
parameters will affect the torsion deformation of waveguide, thus affecting the induced reply signal amplitude of the torsional wave sensing coil. Among the components of torsional wave sensing coil output signal, the induced reply signal represents the useful signal. An increase in the amplitude of induced reply signal can improve the signal to noise ratio of sensing coil output, thus improving the time interval measurement precision. In order to improve the displacement measurement accuracy, experimental study on dependence of the amplitude of torsional wave sensing coil output signal on the width and amplitude of exciting current pulse is carried out in this paper.

Optimization of Excitation Current Pulse Width. Magnetostrictive waveguide used is 3J53 iron nickel alloy wire of diameter $0.5 \mathrm{~mm}$, length $1200 \mathrm{~mm}$. Torsional wave sensing coil output signal waveform for the current pulse width of $7 \mu$ s is shown in Fig. 4 (a), torsional wave sensing coil output signal waveform for the current pulse width of $15 \mu$ s is shown in Fig. 4 (b). Based on the comparison between Fig. 4 (a) and (b), it can be seen, the induced reply signal appears with single peak and double valley when current pulse width is narrow, the induced reply signal appears with double peak and double valley when current pulse width is too wide. For the case of current pulse amplitude $I_{m}=6 \mathrm{~A}$, dependence of the amplitude of torsional wave sensing coil output signal on the width of exciting current pulse is shown in Fig. 5. It can be seen from Fig. 5, as current pulse width increases from $1 \mu \mathrm{s}$, the amplitude of torsional wave sensing coil output signal increases gradually at first, increases to the maximum value at about $7 \mu$ s, and then decreases gradually.

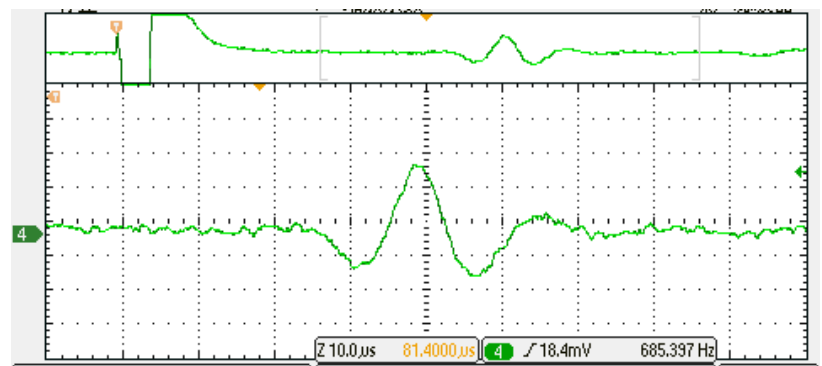

(a)

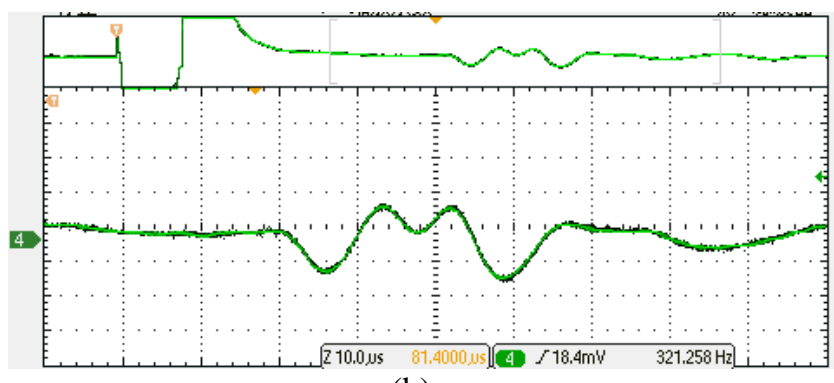

(b)

Fig. 4. The waveform of torsional wave sensing coil output signal

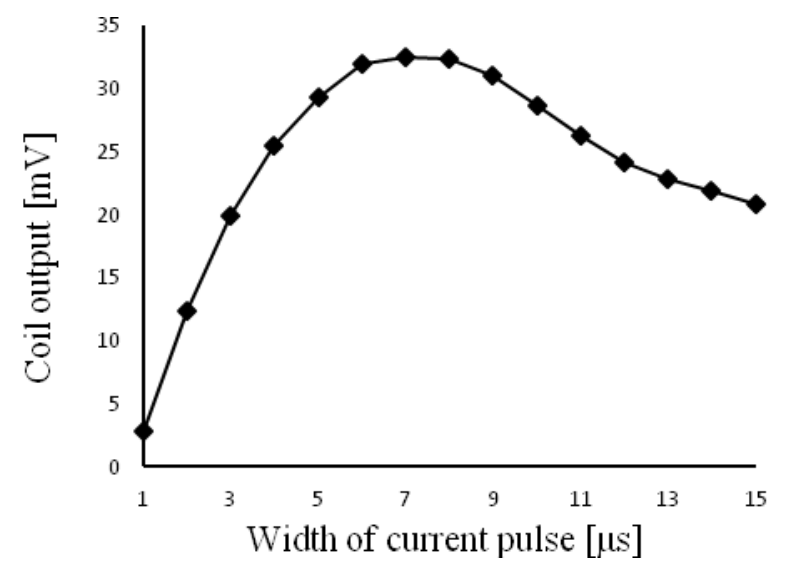

Fig. 5 Dependence of the sensing coil output pulse amplitude on the exciting current pulse width

Optimization of Current Pulse Amplitude. For the case of current pulse width $7 \mu$ s, dependence of the amplitude of torsional wave sensing coil output signal on the current pulse amplitude is shown in Fig. 6. It can be seen from Fig. 6, with the current pulse amplitude increase, the amplitude of torsional wave sensing coil output signal increases more obvious at first, and when the current pulse amplitude exceeds above 6A, the amplitude of torsional wave sensing coil output signal increases slowly. 


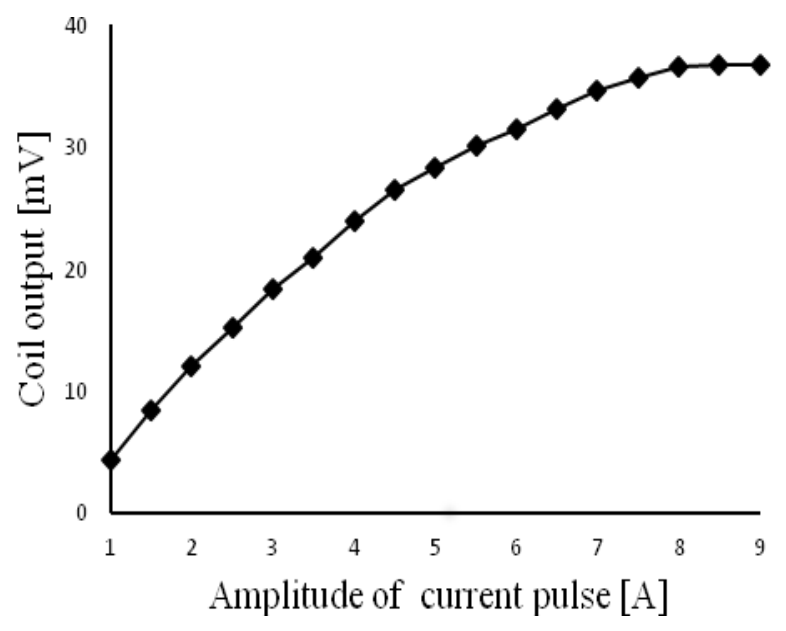

Fig.6 Dependence of the sensing coil output pulse amplitude on the amplitude of the exciting current pulse

\section{Experimental Test}

Experimental Results of Displacement Measurement. The propagation speed in the magnetostrictive waveguide (3J53) of torsional wave is about $2600 \mathrm{~m} / \mathrm{s}$. The exact speed value is related to many factors, such as material composition and heat treatment. We can calculate propagation speed according to the time interval spent when torsional wave propagates through a known distance.

Use vernier caliper to measure the distance of $296.00 \mathrm{~mm}$, measured time interval of the torsional wave through this distance is $115740 \mathrm{~ns}$, so the calculation result of propagation speed of the torsional wave in the waveguide is $2557.4 \mathrm{~m} / \mathrm{s}$.

Displacement measurement experiment is completed in a laboratory environment. In the range of $100 \sim 800 \mathrm{~mm}, 8$ measurement points is measured in an interval of $100 \mathrm{~mm}$. The measurement results are shown in table 1.

Table 1. The results of displacement measurement experiment

\begin{tabular}{|c|c|c|c|c|}
\hline $\begin{array}{c}\text { Displacement } \\
{[\mathrm{mm}]}\end{array}$ & $\begin{array}{c}\text { Measured time } \\
{[\mathrm{ns}]}\end{array}$ & $\begin{array}{c}\text { Computed displacement } \\
\text { value }[\mathrm{mm}]\end{array}$ & $\begin{array}{c}\text { Absolute error } \\
{[\mathrm{mm}]}\end{array}$ & $\begin{array}{c}\text { Rounded absolute } \\
\text { error [mm] }\end{array}$ \\
\hline 100.0 & 39094 & 99.98 & 0.02 & 0.0 \\
\hline 200.0 & 78127 & 199.80 & 0.20 & 0.2 \\
\hline 300.0 & 117179 & 299.67 & 0.33 & 0.3 \\
\hline 400.0 & 156376 & 399.92 & 0.08 & 0.1 \\
\hline 500.0 & 195431 & 499.80 & 0.20 & 0.2 \\
\hline 600.0 & 234590 & 599.94 & 0.06 & 0.1 \\
\hline 700.0 & 273706 & 699.98 & 0.02 & 0.0 \\
\hline 800.0 & 312783 & 799.91 & 0.09 & 0.1 \\
\hline
\end{tabular}

Analysis of Experimental Results. According to the experimental data of Table 1, it can be seen, the sensor error is very small. Because the distance is measured with a ruler, high precision calibration of the transducer can not be carried out. From the measuring data it can be seen that, in the process of measurement the measurement error is within $0.4 \mathrm{~mm}$, the precision of developed magnetostrictive linear displacement transducer can be guaranteed. 


\section{Conclusions}

A new kind of magnetostrictive displacement sensor is developed; the parameters of this transducer are optimized.

(1) For the current pulse amplitude remained unchanged, the current pulse width will have an impact on the amplitude of torsional wave sensing coil output signal. For the waveguide (3J53) of diameter $0.5 \mathrm{~mm}$, when the current pulse width is about $7 \mu \mathrm{s}$, the amplitude of output signal reaches maximum.

(2) For the current pulse width remained unchanged, with the current pulse amplitude increase, the amplitude of torsional wave sensing coil output signal increases more obvious at first, and when the current pulse amplitude exceeds a certain magnitude, the amplitude of output signal increases slowly.

(3) Experimental results show that the precision of developed magnetostrictive displacement sensor can reach less than $1 \mathrm{~mm}$.

\section{Acknowledgements}

This study is supported by the National Natural Foundation of China (No. 61273082 and No. 51271019) and Major Construction Project of Beijing Personnel Training Co-Construction Project (The construction of strategic emerging industries related major).

\section{References}

[1] V. I. Borodin, V. V. Ostanin, S. V. Zhakov: Phys. Met. Metall., 56(5) (1983), p. 96

[2] S. V. Zhakov, V. I. Borodin, V. V. Ostanin: Phys. Met. Metall., 57(1)(1984), p. 45

[3] E. Hristoforou, H. Hauser, A. Ktena: J. Appl. Phys., Vol. 93(2003), p.8633

[4] F. Seco, J. M. Martin, A. R. Jimenez: IEEE Trans. Instrum. Meas., Vol. 58(2009), p. 722

[5] F. Seco, J. M. Martin, A. R. Jimenez, et al: Sensor. Actuat. A-Phys., 123-124(23) (2005), p. 216

[6] K. G. Van den Berg: J. Phys. E: Sci. Instrum., Vol. 15(1982), p.325

[7] A. Affanni, A. Guerra, L. Dallagiovanna, et al: IEEE IMTC2004, Como, Italy, Vol.1 (2004), p. 206

[8] C. Deng , Y. H. Kang, E. L. Li, et al: Measurement., 47(1)(2014), p.591

[9] P. Ferrari, A. Flammini, D. Marioli, et al: IEEE I2MTC2008, Victoria, BC, Canada, (2008), p.1766

[10] C. Deng, Y. H. Kang: Sensor. Actuat. A-Phys., Vol.210(2014), p.99

[11] C. Deng , Y. H. Kang, B. Ye, et al: IEEE T. Instrum. Meas., 63(10)(2014), p.2464

[12] Z. H. Zhou, L. Q. Wang, C. Shen, et al: Chinese J. Sci. Instrum., 35(1)(2014), p.103

[13] T. Xia, X. X. Gao, J. H. Li, et al: J. mag. mater. dev., (4)(2008), p.21

[14] J. H. Li, X. X. Gao, J. Zhu, et al: Chinese Phys. B, 21(2012): 087501

[15] J. H. Li, X. X. Gao, J. Zhu, et al: J. Univ. Sci. Tech. Beijing, Vol.31(2009), p.1281 